\title{
SOME DUAL STATEMENTS CONCERNING WIENER MEASURE AND BAIRE CATEGORY
}

\author{
K. SIMON
}

(Communicated by R. Daniel Mauldin)

\begin{abstract}
This paper deals with the duality between Wiener measure and Baire category on $C_{0}^{1}$, the set of continuous functions $f:[0,1] \rightarrow[0,1]$ endowed with the supremum norm. We prove that some properties shared by a residual set of continuous functions, e.g. the typical level set structure, and the existence of periodic points of order 3 hold a.e. with respect to the Wiener measure.
\end{abstract}

\section{Section 1}

Introduction. This paper deals with the duality between Wiener measure and Baire category on $C_{0}^{1}$, the set of continuous functions $f:[0,1] \rightarrow[0,1]$ endowed with the supremum norm. By the term "typical continuous function" we mean that the set of all functions which have the property under consideration is a residual subset of the complete metric space $C[0,1]$. By the term "almost every continuous function" we mean that the set of all continuous functions which does not have the property under consideration has zero Wiener measure. Let $A$ and $A^{\prime}$ be two statements. If $A^{\prime}$ can be obtained from $A$ by interchanging the terms "typical continuous function" and "almost every continuous function" then $A^{\prime}$ is called the dual of $A$.

It is well known that typical continuous functions have no derivatives, neither finite nor infinite, at any point. The dual statement was proved by Dvoretzky, Erdös and Kakutani [4]. Saks proved in [10] that for a typical continuous function $f$ the set of points at which $f$ has a right-hand side infinite derivative, has cardinality $2^{\mathscr{H}}$ in every interval. We shall prove the dual statement in $\S 3$.

It is proved in [11] that typical continuous functions are not iterates of a continuous function. We shall prove the dual statement in $\S 2$. We also prove that the level set structure of almost every continuous function is the same as the typical level set structure of continuous functions.

It is well known that a typical continuous function is chaotic. We shall prove the dual statement in $\S 4$ and we also prove there that the duality holds in the structure of the set of fixed points.

Received by the editors February 25, 1988 and, in revised form, September 23, 1988.

1980 Mathematics Subject Classification (1985 Revision). Primary 28C20, 54E52; Secondary $60 \mathrm{~J} 65$. 
Notations. We define

$$
\begin{aligned}
C[a, b] & =\{f \mid f:[a, b] \rightarrow R, f \text { is continuous }\}, \\
C & =C[0,1], \\
C_{q}[a, b] & =\{f \in C[a, b] \mid f(a)=q\}, \\
C_{q} & =C_{q}[0,1], \\
C_{0}^{1} & =\{f \in C[0,1] \mid f([0,1]) \subset[0,1]\} .
\end{aligned}
$$

Here $C_{0}^{1}$ is a complete metric space with the supremum norm. Let $\mathscr{B}$ denote the Borel $\sigma$-field of $C_{0}$. A subset $I$ of $C_{0}$ is called a cylinder set, if $I$ is of the following form:

$$
I=\left\{f \in C_{0} \mid\left(f\left(t_{1}\right), \ldots, f\left(t_{n}\right)\right) \in E\right\},
$$

where $0 \leq t_{1}<t_{2}<\cdots<t_{n} \leq 1$ and $E$ is a Borel subset of $R^{n}$. The collection $\mathscr{R}$ of all cylinder subsets of $C_{0}$ is a field. Let $I$ be a cylinder set given above.

Define

$$
\begin{aligned}
P(I) & =\left[(2 \pi)^{n} t_{1}\left(t_{2}-t_{1}\right) \ldots\left(t_{n}-t_{n-1}\right)\right]^{-1 / 2} \\
& \cdot \int_{E} \exp \left\{-\left(\frac{u_{1}^{2}}{t_{1}}+\frac{\left(u_{2}-u_{1}\right)^{2}}{t_{2}-t_{1}}+\cdots+\frac{\left(u_{n}-u_{n-1}\right)^{2}}{t_{n}-t_{n-1}}\right) / 2\right\} d u_{1} \cdots d u_{n} .
\end{aligned}
$$

$P$ has a unique extension, still denoted by $P$, to the Borel $\sigma$-field $\mathscr{B}$. $P$ is called the Wiener measure in $C_{0}$. Let $T_{a}$ denote the map $T_{a}(x)=x+a$ $(x \in R)$. For every $a \in R$ and every Borel subset $H \subset C_{0}[a, a+1]$ we define $P^{(a)}(H)=P\left(H^{\prime}\right)$, where $H^{\prime}=\left\{f \circ T_{a}: f \in H\right\} \subset C_{0}$. Let $B \subset C_{a}$ be a Borel subset, then we define $P_{a}(B):=P_{0}(B-a)$, where $B-a=\left\{f \in C_{0} \mid f+a \in B\right\}$. Using the bijection $\varphi: C_{0} \times R \rightarrow C \varphi((f, a))(x)=f(x)+a$ we shall identify $C$ and $C_{0} \times R$. Let $\mu=P \times m$ where $m$ is the Lebesque measure on $R$. Then $\mu$ is Borel measure on $C=C_{0} \times R$. Let $D$ be a Borel subset of $C_{0}^{1}$ then we put $\nu(D)=\mu(D) / \mu\left(C_{0}^{1}\right)$. Then $\nu$ is a probability measure on $C_{0}^{1}$ because $P\left(\left\{f \mid f\left([0,1]^{\prime}\right) \subset(-\varepsilon, \varepsilon)\right\}\right)>0$ for every $\varepsilon>0$ (see [5], p. 115) and this implies that $\mu\left(C_{0}^{1}\right)>0$.

Let $\operatorname{Fix}(f)=\{x: f(x)=x\}$. For $f \in C_{0}^{1}$ and $n \in N$ we define $f^{n}(x)$ by $f^{1}(x)=f(x)$ and $f^{n}(x)=f\left(f^{n-1}(x)\right)$. A function $f \in C_{0}^{1}$ is said to be locally increasing at a point $x \in[0,1]$ if there is a $\delta>0$ such that $f(t) \leq f(x)$ on $(x-\delta, x) \cap[0,1]$ and $f(t) \geq f(x)$ on $(x, x+\delta) \cap[0,1]$. The function $f$ is locally decreasing at $x$ if $-f$ is locally increasing at $x$. We denote $\bar{B}=C_{0}^{1} \backslash B$. If

$$
\limsup _{\substack{t \rightarrow x \\ t>x}} \frac{m((x, x+t) \cap U)}{t}=\liminf _{\substack{t \rightarrow x \\ t>x}} \frac{m((x, x+t) \cap U)}{t}
$$

then this value is called the right-hand Lebesque density of $U$ at $x$ and it is denoted by $d(x, U)$. For $a \in R, B \subset C$ we define $B_{a}=\{f \in B \mid f(0)=a\}$. For every $f \in C$ we denote by $R E_{f}$ the set of points at which $f$ has a 
local extremum. Let $f_{+}^{\prime}(x)$ be the right-hand derivative of $f$ at $x$ and let $\bar{f}^{+}(x)=\lim \sup _{t \rightarrow x, t>x}(f(t)-f(x)) /(t-x)$.

In what follows we shall make use of the following properties of the Wiener measure:

(a) The mapping $f(t) \rightarrow f\left(t+t_{0}\right)-f\left(t_{0}\right),-\infty<t<\infty$ is a measure preserving mapping of $C_{0}$ onto $C_{0}\left[-t_{0}, 1-t_{0}\right]$ for every $-\infty<t_{0}<\infty$ (see [7], Chapter 7.4).

(b) Strong Markov property:

$$
P\left\{f_{\mathfrak{M}}^{+} \in B \mid \mathscr{B}_{\mathfrak{M}+0}\right\}=P_{b}(B), \mathfrak{M}<\infty, b=f(\mathfrak{M}), B \in \mathscr{B} .
$$

Where $f_{\mathfrak{M}}^{+}(t)=f(t+\mathfrak{M})$ and $\mathfrak{M}$ is a Markov time. (See [6], chapter 1.6.)

(c) $P_{0}\{m\{s: f(s) \geq 0, s \leq t\} \leq \theta\}=\frac{2}{\pi} \arcsin \sqrt{\theta / t}$ (see [6], chapter 2.6).

(d) $P_{0}\left\{\max _{0 \leq u \leq t} f(u) \geq a\right\}=2 / \sqrt{2 \pi} \cdot \int_{\sqrt{a / t}}^{\infty} \exp \left(-y^{2} / 2\right) d y$ (see [7], chapter 7.3).

(e) $P_{0}\{f \mid$ there is $t \in(a, b)$ such that $f(t)=0\}=2 / \pi \arccos \sqrt{a / b}$ (see [7], chapter 7.3).

\section{SeCtion 2}

In this section we shall prove that the set of functions $f \in C_{0}^{1}$ for which there exists $\varphi \in C_{0}^{1}$ and $k \geq 2$ such that $f=\varphi^{k}$ has $\nu$-measure zero.

We define

$$
\begin{aligned}
T_{1}= & \left\{f \in C_{0}^{1} \mid \operatorname{Fix}(f) \cap \mathrm{RE}_{f}=\varnothing\right\}, \\
T_{2}= & \left\{f \in C_{0}^{1} \mid f\right. \text { is not locally increasing or decreasing } \\
& \quad \text { at any } x \in[0,1]\}, \\
T_{3}= & \left\{f \in C_{0}^{1} \mid \operatorname{card}\left(E_{\alpha}^{f} \cap \mathrm{RE}_{f}\right) \leq 1 \quad \text { for every } \alpha \in[0,1]\right\}, \\
T= & T_{1} \cap T_{2} \cap T_{3}, \\
W_{k}= & \left\{f \in C_{0}^{1} \mid \exists \varphi \in C_{0}^{1} \text { such that } f=\varphi^{k}\right\} .
\end{aligned}
$$

Theorem 1. $\nu\left(\left\{f \in C_{0}^{1} \mid f=\varphi^{k}, \varphi \in C_{0}^{1}, k \geq 2\right\}\right)=0$.

Proof. It is proved in [11] that $W_{k} \subset \bar{T}=\bar{T}_{1} \cup \bar{T}_{2} \cup \bar{T}_{3}$ so it is enough to prove that $\nu(\bar{T})=0$.

(a) It is proved in [4] that $P\left(C_{0} \backslash T_{2}\right)=0$, so it is easy to see that $\nu\left(\bar{T}_{2}\right)=0$.

(b) We shall prove that $\nu\left(\bar{T}_{3}\right)=0$.

Let $I_{1}$ and $I_{2}$ disjoint intervals such that $I_{i}=\left[a_{i}, b_{i}\right]$ and $b_{1}<a_{2}$. It will be sufficient to show that if

$$
H=\left\{f \in C_{0} \mid \max _{u \in I_{1}} f(u)=\max _{v \in I_{2}} f(v)\right\}
$$

and

$$
K=\left\{f \in C_{0} \mid \max _{u \in I_{1}} f(u)=\min _{v \in I_{2}} f(v)\right\}
$$

then $P(H)=P(K)=0$. 
First we prove that $P(H)=0$. Let $f: C_{0} \rightarrow C_{0}\left[-b_{1}, 1-b_{1}\right]$ and let $(F(f))(x):=f\left(x+b_{1}\right)-f\left(b_{1}\right)$. Then

$$
F(H)=\left\{f \in C_{0}\left[-b_{1}, 1-b_{1}\right] \mid \max _{a_{1}-b_{1} \leq u \leq 0} f(u)=\max _{a_{2}-b_{1} \leq v \leq b_{2}-b_{1}} f(v)\right\}
$$

and because $F$ is a measure preserving transformation (see $\S 1$ ) it will be sufficient to show that $P^{\left(-b_{1}\right)}(F(H))=0$. Since $P^{\left(-b_{1}\right)}$ is probability measure on $C_{0}\left[-b_{1}, 1-b_{1}\right]$ we can regard $\eta=\max _{a_{1}-b_{1} \leq u \leq 0} f(u)$ and $\xi=$ $\max _{a_{2}-b_{1} \leq v \leq b_{2}-b_{1}} f(v)$ as random variables. We have to prove that $P^{\left(-b_{1}\right)}(\eta=$ $\xi)=0$. It will be sufficient to show that $(\eta, \xi)$ is an absolutely continuous random variable. It is easy to see that $\xi$ is independent of $\eta$. Thus

$$
\begin{aligned}
G(x, y) & =P^{\left(-b_{1}\right)}(\xi<x, \eta>y)=P^{\left(-b_{1}\right)}(\eta<y) \cdot P^{\left(-b_{1}\right)}(\xi<x) \\
& =\int_{0}^{y} \frac{2}{\sqrt{2 \pi\left(b_{1}-a_{1}\right)}} \cdot \exp \left(\frac{z^{2}}{2\left(b_{1}-a_{1}\right)}\right) d z \cdot P^{\left(-b_{1}\right)}(\xi<x) .
\end{aligned}
$$

Let

$$
p(x, t)=\frac{1}{\sqrt{2 \pi t}} \cdot \exp \left(-\frac{x^{2}}{2 t}\right)
$$

then

$$
P^{\left(-b_{1}\right)}(\xi<x)=\int_{-\infty}^{x} P^{\left(-b_{1}\right)}\left(\xi<x \mid f\left(a_{2}-b_{1}\right)=u\right) \cdot p\left(u, a_{2}-b_{1}\right) d u
$$

but

$$
\begin{aligned}
& P^{\left(-b_{1}\right)}\left(\xi<x \mid f\left(a_{2}-b_{1}\right)=u\right)=P^{\left(-b_{1}\right)}\left(\max _{0 \leq c \leq b_{2}-a_{2}} f(c)<x-u\right) \\
& =2 \int_{0}^{x-u} p\left(v, b_{2}-a_{2}\right) d v
\end{aligned}
$$

so

$$
P^{\left(-b_{1}\right)}(\xi<x)=\int_{-\infty}^{x} 2 \cdot \int_{0}^{x-u} p\left(v, b_{2}-a_{2}\right) d v \cdot p\left(u, a_{2}-b_{1}\right) d u .
$$

This implies that $P^{\left(-b_{1}\right)}(\xi<x)$ is absolutely continuous thus $G(x, y)$ is also absolutely continuous, and hence $(\eta, \xi)$ is an absolutely continuous random variable and $P^{\left(-b_{1}\right)}(\eta=\xi)=0$. So $P(H)=0$.

The argument above may be applied without any essential alteration for the proof of $P(K)=0$. This easily implies $\nu(H)=\nu(K)=0$.

Let for every $n=1,2, \ldots, H^{n}, K^{n}$ be sets as the $H, K$ above. Then $\bar{T}_{3} \subset \bar{L} \subset \bigcup_{n}\left(H^{n} \cap K^{n}\right)$, where

$$
L=\left\{f \in C \mid \operatorname{Card}\left(E_{f} \cap \mathrm{RE}_{f}\right) \leq 1 \text { for every } \alpha \in R\right\} .
$$

Thus $\nu(\bar{L})=\nu\left(\bar{T}_{3}\right)=0$.

(c) We shall prove that $\nu\left(\bar{T}_{1}\right)=0$. Let $e(b)=\left\{(x, y) \in R^{2} \mid y=x+b\right\}$ and let $B=\left\{(f, b) \in C \times R \mid\right.$ there is $x \in \mathrm{RE}_{f}$ such that $\left.(x, f(x)) \in e(b)\right\}$. 
Then it is easy to see that $B$ is an $F_{\sigma}$ subset of $C \times R$. Let $\mu_{1}=\mu \times m$. Then $B$ is measurable for $\mu_{1}$ so we can use Fubini's Theorem. Let $f \in L$ and $B_{f}=\{b \in R \mid(f, b) \in B\}$. Then $\operatorname{Card}\left(B_{f}\right)=\aleph_{0}$ and so $m\left(B_{f}\right)=0$. If $B^{b}=\{f \in C \mid(f, b) \in B\}$, then from Fubini's Theorem we get that

$$
\mu_{1}(B)=\int_{C} m\left(B_{f}\right) d \mu=\int_{b \in R} \mu\left(B^{b}\right) d m=\int_{L} m\left(B_{f}\right) d \mu=0 .
$$

Thus, for almost every $b \in R \mu\left(B^{b}\right)=0$. It is easy to see that for every $b, c \in$ $R \mu\left(B^{b}\right)=\mu\left(B^{c}\right)$. Thus, for every $b \in R \mu\left(B^{b}\right)=0$. In particular $\mu\left(B^{0}\right)=0$, and $B^{0} \supseteq \bar{T}_{1}$, so

$$
\nu\left(\bar{T}_{1}\right) \leq \frac{\mu\left(B^{0} \cap C_{0}^{1}\right)}{\mu\left(C_{0}^{1}\right)}=0 .
$$

This completes the proof of Theorem 1 .

Corollary 1. $\nu\left(T_{2} \cap T_{3}\right)=1$.

In Bruckner's book [2] it is proved that if $f \in T_{2} \cap T_{3}$ then there exists a dense denumerable subset $S_{f}$ of the interval $\left(m_{f}, M_{f}\right)$ such that the level set $E_{\alpha}$ is

(i) a nowhere dense perfect set when $\alpha \notin S_{f} \cup\left\{m_{f}, M_{f}\right\}$

(ii) a single point when $\alpha=m_{f}$ or $M_{f}$

(iii) of the form $P_{\alpha} \cup\{x\}$ where $P_{\alpha}$ is a nonempty nowhere dense perfect set and $x_{\alpha}$ is isolated in $E_{\alpha}$ when $\alpha \in S_{f}$.

It follows from this result and Corollary 1 that:

Corollary 2. For almost every continuous $f$ the level structure of $f$ has properties (i), (ii), (iii).

\section{Section 3}

In this section we shall prove the dual of the following theorem of Saks.

Theorem (Saks [10]). For a typical continuous function $f$ there exists a set $H$ such that $\operatorname{card}(H)=2^{\aleph_{0}}$ and $f_{+}^{\prime}(x)=\infty$ at each point of $H$.

The dual statement.

Theorem 2. For almost every $f$ there exists a set $H$ such that $\operatorname{card}(H)=2^{\aleph_{0}}$ and $f_{+}^{\prime}(x)=\infty$ at each point of $H$.

It is well known that if $f$ is continuous, $\bar{f}^{+}(x) \geq 0$ holds almost everywhere and the set of points $x$ at which $\bar{f}^{+}(x)=-\infty$ is countable then $f$ is increasing (see [9], 7.3. Theorem). This monotonicity theorem easily implies the following result. 
Theorem. For every continuous $f:[a, b] \rightarrow R$ either there is a set $H \subset[a, b]$ of positive measure on which $\left.f\right|_{H}$ is monotone, or the set of points $x$ at which $f_{+}^{\prime}(x)=\infty$ has cardinality $2^{\aleph_{0}}$ in every interval. (This observation is due to D. Preiss and was communicated to me by M. Laczkovich.)

Thus, if

$$
\begin{gathered}
K=\left\{f \in C \mid \text { there is } U \subset[0, \infty) \text { such that }\left.f\right|_{U}\right. \text { is monotone } \\
\text { increasing and } m(U)>0\}
\end{gathered}
$$

then it will be enough to show that:

Lemma 1. $\mu(K)=0$.

Proof of Lemma 1. Let $f \in K$ and let $x$ be a density point of $U$. Then $d(x, U)=1$ and for each $u \in U \cap[x, \infty), f(u) \geq f(x)$. Thus if

$$
\begin{aligned}
& K_{1}=\{f \in C \mid m(\{x \in[0, \infty) \mid \text { there is } U \subset[x, \infty) \text { such that } \\
& \qquad d(x, U)=1 \text { and for every } u \in U f(u) \geq f(x)\})>0
\end{aligned}
$$

then $K_{1} \supset K$. So it will be sufficient to prove that $\mu\left(K_{1}\right)=0$.

We define $H \subset C \times R$ as

$$
\begin{gathered}
H=\{(f, t) \mid \text { there is } U \subset[t, \infty) \text { such that } d(t, U)=1 \text { and for } \\
\text { every } u \in U f(u) \geq f(t)\} .
\end{gathered}
$$

First we show that $H$ is measurable with respect to $\mu_{1}$. Let $\left\{a_{m}\right\}$ be a fixed sequence increasing to 1 and

$$
Q_{n}^{m}=\left\{(f, t) \mid m(\{v \mid f(v) \geq f(t) \text { and } v \in(t, t+1 / n)\}) \geq a_{m} \cdot 1 / n\right\} .
$$

Let $\left(f_{k}, t_{k}\right) \rightarrow(f, t)$ and $\left(f_{k}, t_{k}\right) \in Q_{n}^{m}$.

Let $V_{k}=\left\{v \mid f_{k}(v) \geq f_{k}(t)\right.$ and $\left.v \in\left(t_{k}, t_{k}+1 / n\right)\right\}$ and let $V=\bigcap_{l=1}^{\infty} \bigcup_{k=l}^{\infty} V_{k}$, then it is easy to see that $m(V) \geq a_{m} \cdot 1 / n$ and for every $v \in V \quad f(v) \geq$ $f(t)$ so that $(f, t) \in Q_{n}^{m}$. Hence, $Q_{n}^{m}$ is closed. One can easily check that $H=\bigcap_{m=1}^{\infty} \bigcup_{k=1}^{\infty} \cap_{n=k}^{\infty} Q_{n}^{m}$. Thus, $H$ is a Borel subset of $C \times R$ and hence is $\mu_{1}$-measurable.

We prove that $\mu_{1}(H)=0$. Let $H^{t}=\{f \mid(f, t) \in H\}, H^{f}=\{t \mid(f, t) \in H\}$ and $t_{0} \in R$ be fixed. The transformation $f(t) \rightarrow f\left(t+t_{0}\right)-f\left(t_{0}\right)$ is measure preserving (see $\S 1$ ) so it will be enough to show that $P(Z)=0$ where $Z=$ $\left\{f \in C_{0} \mid\right.$ there is $U \subset[0, \infty)$ such that $\left.f(U) \subset[0, \infty), d(0, U)=1\right\}$.

Define

$$
Z_{n}^{m}=\left\{f \in C_{0} \mid m(\{v: f(v) \geq 0 \text { and } v \in(0,1 / n)\}) \geq a_{m} \cdot 1 / n\right\} .
$$

Then $Z=\bigcap_{m=1}^{\infty} \bigcup_{k=1}^{\infty} \bigcap_{n=k}^{\infty} Z_{n}^{m}$ and since $P\left(Z_{n}^{m}\right)=(2 / \pi) \arcsin \sqrt{1-a_{m}}$ (see $\S 1)$, it follows that $P\left(\bigcup_{k=1}^{\infty} \cap_{n=k}^{\infty} Z_{n}^{m}\right) \leq(2 / \pi) \arcsin \sqrt{1-a_{m}}$. As, $1-a_{m} \rightarrow 0^{+}$ this implies that $P(Z)=0$ and thus $\mu\left(H^{t_{0}}\right)=0$, for every $t_{0} \in R$. Applying Fubini's Theorem we obtain that $m\left(H_{f}\right)=0$ holds for $\mu$ almost everywhere $f$ and hence $\mu(K)=0$. This completes the proof of Lemma 1. It is easy to see that Lemma 1 and Theorem 1 imply Theorem 2. 


\section{SECTION 4}

In this section we prove that almost every $f \in C_{0}^{1}$ is chaotic. More exactly, we shall prove the stronger statement that almost every $f \in C_{0}^{1}$ are strictly turbulent. Here $f \in C_{0}^{1}$ is called strictly turbulent iff there exist intervals $I_{1}$, $I_{2}$ such that $I_{1} \cap I_{2}=\varnothing$ and $f\left(I_{1}\right) \cap f\left(I_{2}\right) \supset I_{1} \cup I_{2}$.

It is easy to see that if $f$ is strictly turbulent then $f$ has periodic points of order three (see [3], chapter 2), so for each $n \in N, f$ has periodic points of order $n$ (see [8]).

Theorem 3. $f$ is strictly turbulent for almost every $f \in C_{0}^{1}$.

Proof. Let $\left\{b_{n}\right\}$ be a strictly decreasing sequence such that $b_{n} \rightarrow 0^{+}, b_{n+1} / b_{n}$ $\rightarrow 0$ and $b_{n} / \sqrt{b_{n+1}} \rightarrow 0$ (Take, e.g. $\left.b_{n}=\frac{1}{n !}\right)$. Let

$$
\begin{gathered}
F=\{f \in C \mid \text { there are infinitely many } n \in N \text { such that } \\
\qquad \begin{array}{c}
b_{n}<\max _{0 \leq u \leq b_{n+1}} f(u)-f(0)<\sqrt{b_{n}} \text { and there exists } \\
\left.t \in\left(b_{n+1}, b_{n}\right) \text { such that } f(t)=f(0)\right\} .
\end{array}
\end{gathered}
$$

We prove $\mu(C \backslash F)=0$. It will be enough to show that $P\left(F_{0}\right)=1$. We define

$$
\begin{aligned}
& R_{n}=\left\{\left.f \in C_{0}\right|_{0 \leq u \leq b_{n+1}} f(u) \geq b_{n}\right\}, \\
& Q_{n}=\left\{\left.f \in C_{0}\right|_{0 \leq u \leq b_{n+1}} f(u)<\sqrt{b_{n}}\right\}, \\
& S_{n}=\left\{f \in C_{0} \mid \text { there is } t \in\left(b_{n+1}, b_{n}\right) \text { such that } f(t)=0\right\} .
\end{aligned}
$$

Then $F_{0}=\bigcap_{k=1}^{\infty} \bigcup_{n=k}^{\infty}\left(R_{n} \cap S_{n} \cap Q_{n}\right)$ because

$$
\begin{aligned}
& P\left(R_{n}\right)=\frac{2}{\sqrt{2 \pi}} \int_{b_{n} / \sqrt{b_{n+1}}}^{\infty} \exp \left(-\frac{y^{2}}{2}\right) d y, \\
& P\left(Q_{n}\right)=1-\frac{2}{\sqrt{2 \pi}} \int_{\sqrt{b_{n}} / \sqrt{b_{n+1}}}^{\infty} \exp \left(-\frac{y^{2}}{2}\right) d y, \\
& P\left(S_{n}\right)=\frac{2}{\pi} \arccos \sqrt{\frac{b_{n+1}}{b_{n}}} \quad(\text { see } \S 1) .
\end{aligned}
$$

Thus, $\lim _{n \rightarrow \infty} P\left(R_{n}\right)=\lim _{n \rightarrow \infty} P\left(Q_{n}\right)=\lim _{n \rightarrow \infty} P\left(S_{n}\right)=1$. Consequently, $P\left(F_{0}\right)=1$, and thus $\mu(C \backslash F)=0$. Let $\mathfrak{M}(f)=\min \{u: f(u)=u\}$. Then for each $a \in R \mathfrak{M}$ is a Markov time on $\left(C_{a}, P_{a}\right)$. Let

$$
\begin{gathered}
E=\left\{f \in C_{0}^{1} \mid \text { there is } 0<t<1-\mathfrak{M}(f) \text { such that } \mathfrak{M}=f(\mathfrak{M})\right. \\
\left.=f(\mathfrak{M}+t), \max _{\mathfrak{M} \leq u \leq \mathfrak{M}+t} f(u)>\mathfrak{M}+t\right\}
\end{gathered}
$$

It is easy to see that if $f \in E$ then $f$ is strictly turbulent and $\nu(E)=1$. Indeed, for every $a \in R$ the Brownian motion has the strong Markov property (see $\S 1$ ) 
and so $P_{a}\left(\left(C_{a} \backslash E\right)_{a}\right)=P_{f(\mathfrak{M})}\left(f_{\mathfrak{M}}^{+} \in(C \backslash F) \mid B_{\mathfrak{M}+0}\right)=0$. Thus $\mu(C \backslash E)=0$, $\nu(E)=1$ and this completes the proof of Theorem 3 .

From Lemma 1 is follows that if $f \in C_{0}^{1}$ then $\operatorname{Fix}(f)$ has zero Lebesque measure for $\nu$-almost every $f \in C_{0}^{1}$. We now prove:

Theorem 4. For $\nu$-almost every $f \in C_{0}^{1} \operatorname{Fix}(f)$ is a nowhere dense, perfect and zero measure subset of $[0,1]$.

Proof. Since every $f \in C_{0}^{1}$ is increasing on the set $\operatorname{Fix}(f)$ it follows from Lemma 1 that $m(\operatorname{Fix}(f))=0$ for almost every $f$. Since $\operatorname{Fix}(f)$ is closed for all $f \in C_{0}^{1}$ it will be sufficient to show that $\operatorname{Fix}(f)$ has no isolated point for $\nu$-almost every $f \in C_{0}^{1}$. It is enough to prove that for every fixed $t_{1}, t_{2} \in(0,1)$ the set

$$
K=\left\{f \in C \mid \operatorname{card}\left\{t \in\left(t_{1}, t_{2}\right) \mid f(t)=t\right\}=1\right\},
$$

has $\nu$-measure zero.

Let $\mathfrak{M}^{\prime}(f)=\min \left\{u>t_{1} \mid f(u)=u\right\}$. Then $\mathfrak{M}^{\prime}$ is Markov time. The strong Markov property implies that for each $a \in R$

$$
P_{a}\left(\mathfrak{M}^{\prime}<t_{2}, f^{+} \in F\right)=E_{a}\left(\mathfrak{M}^{\prime}<t_{2}, P_{f(\mathfrak{M})}(F)\right)=P_{a}\left\{\mathfrak{M}^{\prime}<t_{2}\right\}
$$

and this implies that $\nu(K)=0$.

In [1] it is proved that for a typical continuous $f, \operatorname{Fix}(f)$ is also a nowhere dense perfect, zero measure subset of $[0,1]$.

\section{ACKNOWLEDGMENT}

The author would like to thank Professor Miklós Laczkovich for his useful advice.

\section{REFERENCES}

1. S. J. Agronsky, A. M. Bruckner and M. Laczkovich, Dynamics of typical continuous functions (to appear in Journal of the London Mathematical Society).

2. A. M. Bruckner, Differentiation of real functions, Lect. Notes in Math. 659, Springer-Verlag, Berlin, 1978.

3. W. A. Coppel, Iterates of continuous maps of an interval into itself, Lectures Notes in Math., 1984.

4. A. Dvoretsky, P. Erdös and S. Kakutani, Nonincreasing everywhere of the Brownian motion process, 4th Proceedings of the Berkeley Symposium on Mathematical Statistics and Probability II, Univ. of California Press, 1961, pp. 103-106.

5. Hui-Hsiung Kuo, Gaussian measures in banach spaces, Lect. Notes in Math. 463, SpringerVerlag, Berlin, 1975.

6. K. Ito and H. P. McKean, Diffusion processes and their sample paths, Springer-Verlag, Berlin, 1965.

7. S. Karlin and H. M. Taylor, A first course in stochastic processes, Academic Press, New York, 1975.

8. T. Li and J. A. Yorke, Period three implies chaos, Amer. Math. Monthly (1975) (82), 985-992.

9. S. Saks, Theory of the integral, Monograf. Mat. 7, Warsaws-Lwow, (1937). 
10. S. Saks, On the functions of Besicovitch in the space of continuous functions, Fund. Math. 19 (1932), 211-219.

11. K. Simon, Typical continuous functions are not iterates (to appear).

Department of Mathematics, Technical University, Miskolc, Hungary 